Palabras clave: Síntomas negativos, Síntomas positivos, Esquizofrenia, Estado de ansiedad, Rasgo de ansiedad.

\title{
Ansiedad-Estado en la Esquizofrenia
}

\author{
Yasuhiro Kaneda, MD, PhD*,** \\ Isao Nagamine, $\mathrm{MD}, \mathrm{PhD}^{*}$ \\ Akira Fujii, MD** \\ * Departamento de Neuropsiquiatría, \\ Universidad de Tokushima. Facultad de \\ Medicina \\ ** Departamento de Neuropsiquiatría, \\ Hospital de Fujii \\ JAPÓN
}

\begin{abstract}
RESUMEN - Objetivo: Se trata de evaluar el estado de ansiedad, en pacientes esquizofrénicos mediante el Inventario de Estado-Rasgo de Ansiedad de Spielberger.

Material: Los sujetos, que forman parte del estudio son un total de 101 pacientes ingresados y diagnosticados mediante el DSM-IV de esquizofrenia.

Resultados: 1) No se han encontrado diferencias significativas, con respecto a la media total de entre los pacientes esquizofrénicos y la población normal con respecto al sexo. 2) La media total de la puntuación del rasgo de ansiedad, fue significativamente más alta en los pacientes esquizofrénicos que en la población sana, en el caso de los hombres pero no así en la mujeres. 3) En los pacientes del sexo masculino se encontró una correlación positiva entre la puntuación total del estado de ansiedad y la puntuación de los síntomas positivos, a diferencia de lo que sucede en las mujeres. 4) En los varones con esquizofrenia existe una correlación positiva significativa, entre la puntuación total de rasgo de ansiedad y las puntuaciones de los síntomas positivos, lo cual no se observó en las mujeres con esquizofrenia.

Conclusiones: El resultado de este estudio demuestra que las mujeres diagnosticadas de esquizofrenia, se muestran biológicamente insensibles al estrés, comparado con los varones.
\end{abstract}

\section{Introducción}

Han aumentado los estudios sobre la comorbilidad de trastornos psiquiátricos, ya que ellos pueden ejercer una significativa influencia sobre el que se presume es el diagnóstico fundamental (Coryell \& Zimmerman 1988, Lesser, Rubin 1988). Tanto los síntomas de ansiedad, como los de depresión están presentes frecuentemente en la mayoría de los trastornos psiquiátricos. 
De todas formas no existen muchos estudios sobre la presencia de síntomas de ansiedad llevados a cabo entre los pacientes esquizofrénicos a diferencia de los estudios sobre síntomas depresivos. Hemos investigado el nivel de estado-rasgo de ansiedad en pacientes esquizofrénicos así como las asociaciones existentes entre ansiedad y síntomas psicóticos.

\section{Material y método}

El estudio se ha llevado a cabo en 54 personas que formaban parte del grupo control, (compuesto de 29 mujeres y 25 hombres) y en 101 pacientes ingresados, diagnosticados de esquizofrenia y en tratamiento desde hace años (43 mujeres y 58 hombres). El diagnóstico se estableció mediante el DSMIV (Asociación Psiquiátrica Americana 1994) considerando así el cumplimiento de los criterios diagnósticos para la esquizofrenia. Para ello se tuvieron en cuenta los datos obtenidos de la entrevista clínica así como la revisión de su historia psiquiátrica.

El estudio fue aprobado por el comité ético y desarrollado de acuerdo con la declaración de Helsinki II. Se obtuvo el consentimiento informado de los sujetos que participaron en el estudio. La tabla I nos muestra las características demográficas de los sujetos. No existían alteraciones tanto en la función renal como en la hepática y se excluyó de la investigación a aquellos pacientes que presentaban cualquier otro tipo de patología del sistema nervioso central, el abuso de tóxicos o un retraso mental. Por otro lado los sujetos del grupo normal no debían seguir ningún tipo de tratamiento farmacológico.
Los niveles de ansiedad se evaluaron mediante la versión japonesa (Nakazato \& Mizuguchi 1982) del Inventario de Ansiedad Estado-Rasgo de de Spielberger (STAI) (Spielberger, Gorsuch 1970). El STAI está formado por dos escalas de 20 ítems construidas para medir el estado y rasgo de ansiedad utilizando para ello escalas de cuatro puntos. Las puntuaciones del STAI se calcularon para el total de los 40 ítems. Antes del estudio se estableció la validez concurrente de la STAI para la esquizofrenia.

Los síntomas psicóticos se definieron empleando la Escala Breve para la Evaluación Psiquiátrica (BPRS) (Overall\& Gorham 1962). La BPRS viene a ser un método estandarizado que consta de 18 síntomas psiquiátricos a los que se aplica una escala de hasta 7 puntos. Dos síntomas las alucinaciones y los trastornos del contenido del pensamiento, derivan de la Escala de Síntomas Positivos basada en los grupos de síntomas de la BPRS (Crow 1985, Guy 1976, Kitamura, Shima 1989, Nicholson, Chapman 1995). La puntuación de los síntomas positivos se estableció realizando un sumatorio de estas escalas. Tres síntomas, la emotional withdrawal, el enlentecimiento motor y el embotamiento afectivo provenían de la escala de síntomas negativos (Crow 1985, Guy 1976, Nicholson, Chapman 1995). La puntuación de los síntomas negativos se estableció mediante la suma de estos.

El análisis estadístico se realizó mediante el empleo de los test paramétricos (el t-test de Student, el coeficiente de correlación de Pearson) con objeto de evaluar los datos si éstos seguían una distribución normal. En otros casos se emplearon tests no paramétricos (U de Mann Witney's y el coeficiente de correlación de Spearman). 


\section{Resultados}

\section{Validación del STAI en esquizofrenia:}

Para la validación concurrente del STAI en esquizofrenia, las puntuaciones de la ansiedad-estado en el STAI estaban correlacionadas con la puntación de los síntomas de ansiedad en el BPRS en 43 pacientes. Existía una correlación positiva entre ellos (Spearman $\mathrm{p}=, 222, \mathrm{p}<, 05, \mathrm{~N}=43$ ).

La puntuación de la ansiedad-rasgo se valoró en 43 pacientes mediante la nueva realización del test tras un periodo de 83,2 días de media ( $S D=44,3$, rango: $13-196)$. La correlación de rango de Spearman mostraron una alta y significativa correlación entre ambos resultados $(\mathrm{p}=, 796, \mathrm{p}<, 0001, \mathrm{~N}=43)$

\section{Ansiedad en pacientes esquizofrénicos}

La tabla I muestra la puntuación total con respecto a la ansiedad-estado en sujetos nor- males y en enfermos esquizofrénicos. No existían diferencias significativas en la media total de la puntuación ansiedad-estado entre ambos grupos de cada sexo.

La tabla I también nos presenta las puntuaciones totales de la ansiedad-rasgo en sujetos normales y pacientes esquizofrénicos. La media total de la ansiedad-rasgo en los pacientes puntuó significativamente en los pacientes esquizofrénicos frente a los varones sanos (U-test, $\mathrm{p}<, 01)$ sin embargo no se dieron diferencias significativas en el caso de las mujeres.

\section{Relación entre ansiedad y los síntomas de esquizofrenia}

La figura 1 muestra la relación existente entre la puntuación total de la ansiedadestado y la puntuación de los síntomas positivos. Son los hombres los que presentan una correlación positiva (Spearman $\mathrm{p}=, 343$, $\mathrm{p}<, 01, \mathrm{~N}=58$ ). Entre las mujeres sin embargo no se encontró una correlación significativa.

Tabla I

Características demográficas de los individuos

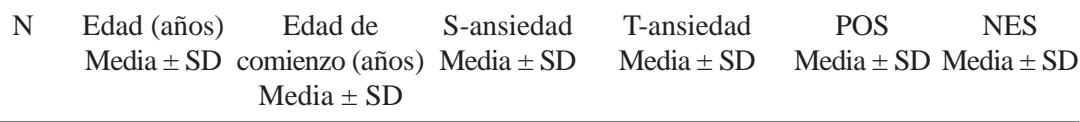

\begin{tabular}{llllllll}
\hline Esquizofrénicos & & & & & & \\
$\quad$ Mujeres & 43 & $49,5 \pm 12,3$ & $30,9 \pm 9,4$ & $42,4 \pm 11,8$ & $43,9 \pm 11,2$ & $5,2 \pm 3,3$ & $6,2 \pm 2,8$ \\
Hombres & 58 & $47,2 \pm 10,8$ & $28,3 \pm 19,7$ & $45,6 \pm 11,1$ & $47,2 \pm 10,8$ & $4,6 \pm 2,6$ & $6,9 \pm 2,7$ \\
Normales & & & & & & & - \\
Mujeres & 29 & $45,1 \pm 9,2$ & - & $41,8 \pm 9,0$ & $44,0 \pm 9,4$ & - & - \\
Hombres & 25 & $33,6 \pm 7,9$ & - & $41,4 \pm 8,4$ & $40,7 \pm 7,7$ & - & - \\
\hline
\end{tabular}

NES = Síntoma negativo.

Nor $=$ Sujetos normales.

POS = Síntoma positivo.

$\mathrm{Sch}=$ Esquizofrénicos.

S-ansiedad = Estado-ansiedad.

T-ansiedad $=$ Ansiedad-rasgo.

(*) $\mathrm{p}<, 05$

$(* *) \mathrm{p}<, 01$

$(* * *) \mathrm{p}<, 001$ frente a normales por el Mann Whitney's U-test. 


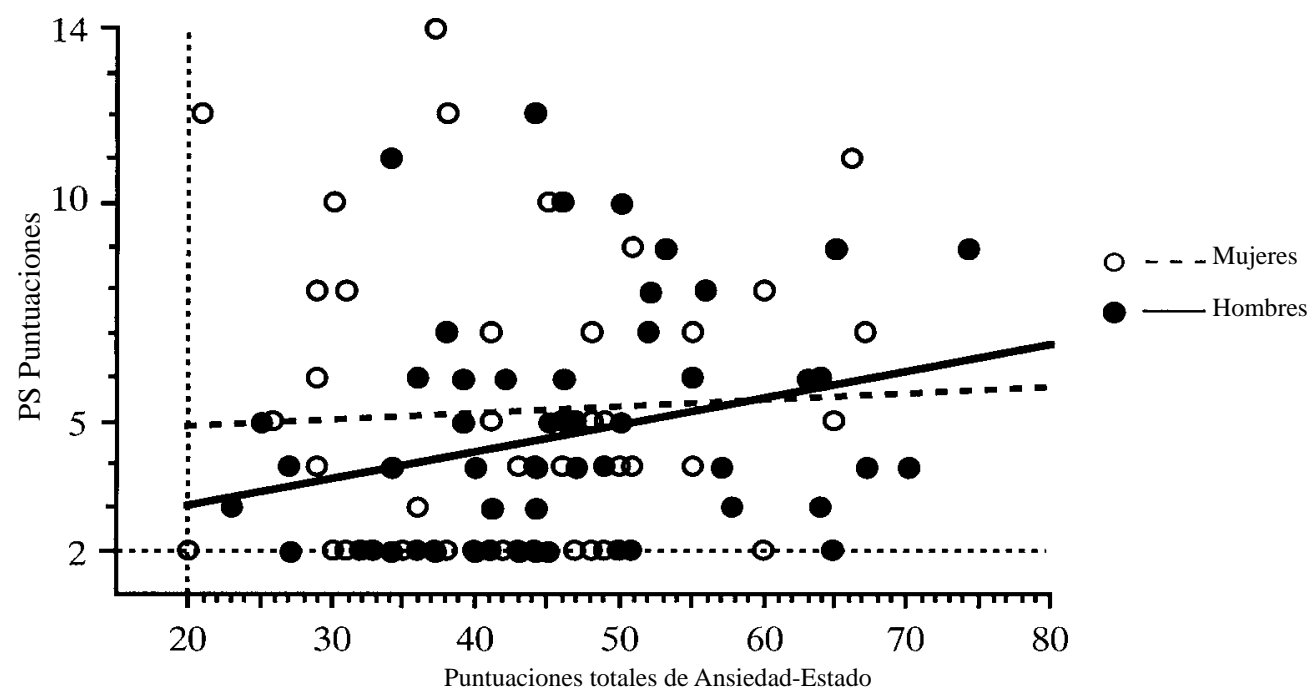

Figura 1. Relación entre las puntuaciones totales de la ansiedad-estado y las de los síntomas positivos (Ps, puntuaciones). En los hombres esquizofrénicos existe una correlación positiva significativa (Spearman $\mathrm{p}=, 343, \mathrm{p}<, 01, \mathrm{~N}=58$ ), pero ello no se dio entre las mujeres con esquizofrenia.

Tampoco existió correlación significativa en la puntuación total de la ansiedad-estado y los valores de los síntomas negativos obtenidos en los pacientes según el sexo.
La figura 2 nos muestra la relación existente entre la puntuación total de la ansiedad-rasgo y la puntuación de los síntomas positivos. En el caso de los hombres existía una correlación

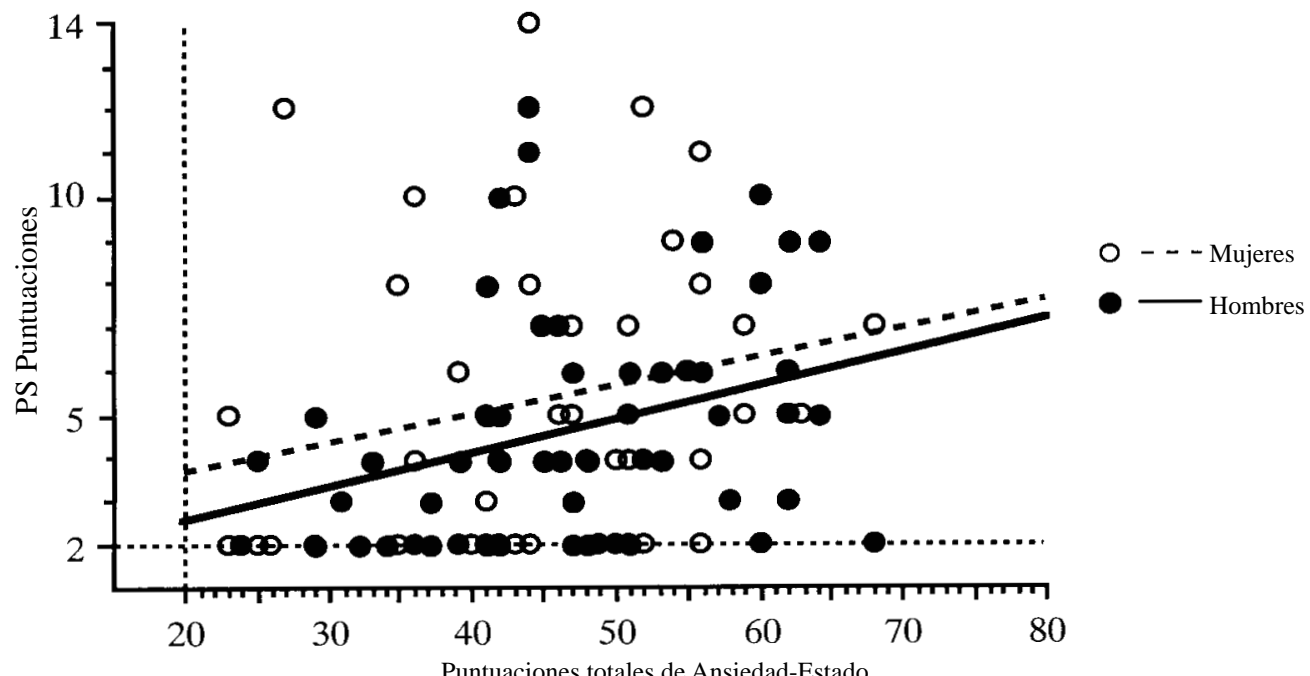

Figura 2. Relación entre las puntuaciones totales de la ansiedad-rasgo y las puntuaciones de los síntomas positivos (PS). En el caso de los hombres con esquizofrenia se da una correlación positiva significativa (Spearman $\mathrm{p}=, 363, \mathrm{p}<, 01, \mathrm{~N}=$ 58). Entre las mujeres con esquizofrenia, las enfermas con valores más altos en las puntuaciones de los síntomas positivos (PS) tienden a presentar igualmente puntuaciones totales altas en la ansiedad-rasgo. 
positiva significativa (Spearman $\mathrm{p}=, 363$, $\mathrm{p}<, 01, \mathrm{~N}=58$ ). Entre las pacientes a pesar de no existir una correlación positiva, se observaba que aquellas que puntuaban más alto en los síntomas positivos, solían presentar también valores altos en la escala de ansiedad-rasgo.

No existía correlación significativa entre la puntuación total de la ansiedad-rasgo y la de los síntomas negativos para los pacientes de ambos sexos. Tampoco se encontraron resultados significativos entre la puntuación total de la ansiedad-rasgo y los periodos de morbilidad para los pacientes de ambos sexos.

\section{Relación entre la ansiedad y la edad de los sujetos sanos}

No se observó correlación significativa entre la puntuación total de la ansiedadestado y la edad para los sujetos normales de cada sexo.

En el caso de las mujeres del grupo control, si que existía una correlación negativa significativa entre la puntuación de la ansiedad-rasgo y la edad (Spearman p =,404, $\mathrm{p}<, 05, \mathrm{~N}=29)$. Entre los hombres del grupo control no se encontraron correlaciones significativas entre ellos.

\section{Discusión}

En primer lugar, hemos de decir que el STAI es una escala de sencilla aplicación para conocer el componente de ansiedad en la esquizofrenia.

Strian y Klicpera (1983) obtuvieron en un grupo de pacientes esquizofrénicos ingresados, un mayor índice de ansiedad frente a otro grupo compuesto por personas sanas, sin embargo en el momento del alta los nive- les de ansiedad eran parecidos a los del grupo de personas sanas. También estos autores señalaron correlaciones entre síntomas de ansiedad y síntomas paranoides. Además, Broman et al. (1995) establecieron que los hombres presentaban una mayor asociación de distrés con disforia que lo que sucedía en el caso de las mujeres. En este estudio, no se observaron diferencias significativas en cuanto al nivel de ansiedad, entre pacientes esquizofrénicos y personas sanas.

De todas formas, el nivel de la ansiedadestado en los hombres esquizofrénicos estaba correlacionado con la gravedad de los síntomas positivos y no con la de los síntomas negativos, con lo que nuestros resultados coinciden parcialmente con los de Strian y Klicpera (1983) y los de Broman et al. (1995)

En la mayoría de los pacientes del sexo masculino, el nivel de la ansiedad-rasgo fue significativamente mayor entre los pacientes con esquizofrenia que entre los sujetos del grupo normal. Las diferencias de edad existentes entre los pacientes y los sujetos sanos no pudieron explicar esta diferencia, tal y como no solo muestra esta investigación sino también estudios previos (Nakazato \& Shimonaka 1989), en los que se vio como los individuos de mayor edad presentaban niveles de ansiedad superiores a los de los jóvenes. Además el nivel de ansiedad-rasgo estaba correlacionado con la gravedad de los síntomas positivos, pero no con la de los síntomas negativos ni tampoco con los periodos de normalidad. Como conclusión de los datos obtenidos, podríamos decir que la ansiedad es más una manifestación que una causa de esquizofrenia. De todas maneras la cuestión todavía queda en el aire, en torno a si un alto nivel de ansiedad-rasgo puede ser un factor de riesgo para el padecimiento de la esquizofrenia. Para aclarar este punto sería preciso, la realización de estudios de tipo prospectivo. 
En nuestro estudio, las diferencias entre los hombres y las mujeres con esquizofrenia se observaron en cuanto a las relaciones entre ansiedad y esquizofrenia: la media total de la puntuación de la ansiedad-rasgo fue significativamente mayor entre los pacientes esquizofrénicos que entre los sujetos normales, aunque solamente se hallaron estas diferencias en el caso de los hombres. A pesar de ello, la gravedad de los síntomas psicóticos, no pueden explicar estas diferencias mientras no se obtenga significación con respecto al sexo, en cuanto a la gravedad de los síntomas psicóticos.

En general, la activación neuroendocrina como repuesta al estrés, en las mujeres suele ser mayor que la que se da entre los hombres (Jezova et al. 1996) y también la experiencia estresante es mayor entre las mujeres bajo las mismas condiciones (Clark \& Rieker 1986, Jones 1993). De hecho nuestros datos acerca de los sujetos sanos y los de anteriores estudios (Nakazato \& Shimonaka 1989) demuestran, que las mujeres tienden a tener niveles más altos de ansiedad que los hombres.

En los últimos años se ha prestado un especial interés, al papel que juega la serotonina (5HT) y otra serie de neurotrasmisores implicados en la fisiopatología de los trastornos de ansiedad, así como en el tratamiento de estos cuadros clínicos mediante los modernos antipsicóticos, con especial atención al papel de la 5HT en la esquizofrenia (Abi-Dargham et al. 1997, Breier 1995, Wooley \& Shaw 1954). También se sugiere la posibilidad de que la colecistoquinina (CCK), sea un péptido implicado en la neurobiología de la ansiedad (Bradwejn \& de Montigny 1984, de Montigny 1989), y también que la disminución de la actividad colecistoquinérgica, aparezca en los pacientes esquizofrénicos (Ferrier et al. 1985, Virgo et al. 1995). A la vista de todos estos descubrimientos pode- mos decir, que las mujeres que padecen de esquizofrenia parecen ser biológicamente insensibles al estrés, comparado con lo que sucede entre los hombres con esquizofrenia. Las diferencias de sexo en la esquizofrenia ya habían sido estudiados previamente (Cowell et al. 1996), y nuestros resultados añaden nuevas evidencias a los estudios anteriores.

\section{Conclusiones}

La media total de las puntuaciones de la ansiedad-rasgo fue significativamente mayor entre los pacientes esquizofrénicos del sexo masculino que entre los sujetos sanos. Además se vio una significativa correlación positiva en estos pacientes, en lo que respecta a la valoración de los síntomas positivos y la de la ansiedad-rasgo y estado. Estos resultados parecen indicar que las mujeres que padecen de esquizofrenia son biológicamente insensibles al estrés comparado ello con los pacientes esquizofrénicos del sexo masculino.

\section{Reconocimientos}

Los autores de éste estudio desean agradecer, los comentarios del profesor Tetsuro Ohmori (Tokushima, Japón) y la apreciable colaboración realizada por el equipo de nuestro Departamento.

\section{Bibliografía}

ABI-DARGHAM, A., LARUELle, M., AGHAJANIAN, G.K., et al. The role of serotonin in the pathophysiology and treatment of schizophrenia. Journal of Neuropsychiatry and Clinical Neurosciences, 9: 1-17, 1997. 
American Psychiatric Association. Diagnostic and Statistical Manual of Mental Disorders, Fourth Edition (DSM-IV), Washington, D.C., American Psychiatric Association, 1994.

BRADWEJN, J., DE MONTIGNY, C. Beuzodiazepines antagonize cholecystokinin-induced activation of rat hippocampal neurones. Nature, 312: 363-364, 1984.

BREIER, A. Serotonin, schizophrenia and antipsychotic drug action. Schizophrenia Research, 14: 187-202. 1995.

BROMAN, C.L., HAMILTON, V.L., HOFFMAN, W.S., et al. Race, gender, and the response to stress: autoworkers' vulnerability to long-term unemployment. American Journal of Community Psychology, 23: 813-842. 1995.

CLARK, E.J., RIEKER, P.P. Gender differences in relationships and stress of medical and law students. Journal of Medical Education, 61: 32-40, 1986.

CORYELL, W., ZIMMERMAN, M. Diagnosis and outcome in schizo-affective depression: a replication. Journal of Affective Disorders, 15: 21-27, 1988.

COWELL, P.E., KOSTIANOVSKY, D.J., GUR, R.C., et al. Sex differences in neuroanatomical and clinical correlations in schizophrenia. The American Journal of Psychiatry, 153: 799-805, 1996.

CROW, T.J. The two-syndrome concept: origins and current status. Schizophrenia Bulletin, 11: 471-486, 1985.

DE MONTIGNY, C. Cholecystokinin tetrapeptide induces panic-like attacks in healthy volunteers. Preliminary findings. Archives of General Psychiatry, 46: 511-517, 1989.

FERRIER, I.N., CROW, T.J., FARMERY, S.M., et al. Reduced cholecystokinin levels in the limbic lobe in schizophrenia. A marker for pathology underlying e defect state? Annals of the New York Academy of Sciences, 448: 495-506, 1985.

GUY, W. ECDU assessment manual, pp. 157-171. Rockville, US Department of Health Education and Welfare, 1976.

JEZOVA, D., JURANKOVA, E., MOSNAROVA, A., et al. Neuroendocrine response during stress with relation to gender differences. Acta Neurobiologiae Experimentalis, 56: 779-785, 1996.

JONES, R.W. Gender-specific differences in the perceived antecedents of academic stress. Psychological Reports, 72: 739-743, 1993.

KITAMURA, T., SHIMA, S., KATO, M., et al. Positive and negative symptoms of chronic schizophrenia (in Japa- nese). Seishin Igalu (Clinical Psychiatry), 31: 131-136, 1989.

LESSER, I.M., RUBIN, R.T., PECKNOLD, J.C., et al. Secondary depression in panic disorder and agoraphobia. I. Frequency, severity, and response to treatment. Archives of General Psychiatry, 45: 437-443, 1988.

LOTSTRA, F., VERBANCK, P.M., GILLES, C., et al. Reduced cholecystokinin levels in cerebrospinal fluid of parkinsonian and schizophrenic patients. Effect of ceruletide in schizophrenia. Annals of the New York Academy of Sciences, 448: 507-517, 1985.

NAKAZATO, K., MIZUGUCHI, T. Development and validation of Japanese version of State-Trait Anxiety Inventoy: a study with female subjects (in Japanese). Shishin Igaku (Japanese Journal of Psychosomatic Medicine), 22: 107-112, 1982.

NAKAZATO, K., SHIMONAKA, Y. The Japanese State-Trait Anxiety Inventory: age and sex differences. Perceptual and Motor Skills, 69: 611-617, 1989.

NICHOLSON, I.R., CHAPMAN, J.E., NEUFELD, R.W. Variability in BPRS definitions of positive and negative symptoms. Schizophrenia Research, 17: 177-185, 1995.

OVERALL, J.E., GORHAM, D.R. The brief psychiatric rating scale. Psychological Reports, 10: 799-812, 1962.

SPIELBERG, C.D., GORSUCH, R.L., LUSHENE, R.E. STAI manual. California, Consulting Psychologists Press, 1970.

STRIAN, F., KLICPERA, C. Anxiety in schizophrenic psychoses. Archiv Jir Psychiafrie und Nerventrankheiten, 233: 347-357, 1983.

VIRGO, L., HUMPHRIES, C., MORTIMER, A., et al. Cholecystokinin messenger RNA deficit in frontal and temporal cerebral cortex in schizophrenia. Biological Psychiatry, 37: 694-701. 1995.

WOOLEY, D.W., SHAW, E. A biochemical and pharmacological suggestion about certain mental disorders. Proceedings of the National Academy of Sciences of the United States of America, 40: 228-231, 1954.

Dirección para correspondencia:

Yasuhiro Kaneda

Departamento de Neuropsiquiatría

Facultad de Medicina de la Universidad de Tokushima

3-18-15 Kuramoto-Cho

Tokushima 770-8503

E-mail: kaneda@clin.med.tokushima-u.ac.jp JAPÓN 